\title{
SOLUTION OF CERTAIN FUNCTIONAL EQUATIONS RELATIVE TO A GENERAL LINEAR SET*
}

BY

\author{
MARK H. INGRAHAM
}

\section{INTRODUCTION}

This paper exhibits the solution of the functional equation

$$
\sum_{j}^{0, n+1}(-1)_{n+1}^{i} C_{j} f\left(x_{0}+j x_{1}\right)=0
$$

for every $x_{0}$ and $x_{1}$ belonging to a linear set of a certain type. As is well known, relative to the complex, real or rational number system any polynomial of degree $n$ satisfies $(A)$. Equation $(A)$ simply states that the $(n+1)$ st difference of the function $f$ vanishes for every set of values of the argument with a constant difference.

Both the equation studied and the method pursued are generalizations of the equation and method of Legendre, $\uparrow$ Hamelf and Schimmack $\S$ in their studies of the equation

$$
f(x+y)=f(x)+f(y) .
$$

It is obvious that any solution of (1) is also a solution of $(A)$ for the case in which $n=1$.

Hamel shows that if we assume that the system $\Re$ of real numbers can be normally ordered (well ordered) then a set $\Re_{0}$ of real numbers exists, such that all elements of $\Re_{0}$ are linearly independent as to finite combinations with rational coefficients and such that every real number is a linear combination of a finite number of elements of $\Re_{0}$ with rational coefficients.

Consider $\Re_{0}$ as a range and $V$ the set of all vectors with rational coördinates of which, for any one vector, only a finite number are different from zero. It is obvious that the elements of $\Re$ may be set into one to one correspondence with the elements of $V$ in such a way that the correspondence is preserved under addition and multiplication by rational numbers.

Hamel shows that any solution of (1) is a linear combination of the coördinates of the vectors of $V$. This paper shows that as a direct generaliza-

* Presented to the Society, April 11, 1925; received by the editors before December, 1925.

$\dagger$ A. M. Legendre (not verified by direct reference) Eléments de Géométrie, 4th edition, Paris, 1802, p. 279.

$\ddagger$ Hamel, Mathematische Annalen, vol. 60 (1905), p. 459.

$\S$ R. Schimmack, Axiomatische Untersuchungen über die Vektoraddition, Nova Acta, Halle, 1908. 
tion the solution of $(A)$ is a polynomial in the coördinates of the vectors of $V$.

\section{RÉSUMÉ OF CERTAIN RESULTS IN THE THEORY OF LINEAR SETS*}

Consider a number system $\mathfrak{A} \equiv[a]$ which is of such a type $\dagger$ that it permits of unique associative and commutative addition, and of unique associative but not necessarily commutative multiplication and also permits of subtraction and division by all numbers not zero while multiplication is distributive as to addition. Any field is such a system, likewise the totality of all real quaternions forms such a system. Such a system $\mathfrak{A}$ we say is of type $A$.

Consider $\mathfrak{X}^{\prime}=\left[\right.$ all $\left.\left.a \cdot \exists \cdot a_{1}.\right) \cdot a a_{1}=a_{1} a\right]$, $\neq$ i. e., the totality of all members of $\mathfrak{A}$ which are commutative as to multiplication with every member of $\mathfrak{A}$. Then $\mathfrak{A}^{\prime}$ is a field. $\S$

Every field contains a sub-field $R$ isomorphic with the field of all rational numbers or the field of all integers modulo a prime, $p$. In the first case we will say that $R$ and consequently $\mathfrak{A}$ and $\mathfrak{A}^{\prime}$ have the modulus $\omega$, otherwise the modulus $p$. The systems $R$ and $\mathfrak{A}$ form an example of a system $\mathfrak{A}$ of type $A$ and an abstract set of vectors $\| U$ connected by a unique associative and commutative addition process and connected with $\mathfrak{A}$ by a process of scalar multiplication which is unique, associative and distributive with respect to addition but not necessarily commutative. In this particular case scalar multiplication is commutative.

If we assume that $U$ has a normally ordered base $U_{1}$ in terms of the elements of which any element of $U$ is expressible as a finite linear combination with coefficients in $R$, it can be shown that there exists a base $U_{0}$ having the same property and such that its terms are finitely linearly independent as to coefficients in $R$. In such a case $U$ is isomorphic with the totality $V=[v]$ of vectors on $U_{0}$ to $R$ finitely non-zero. Of course, if $U$ is normally ordered $U$ itself is effective as $U_{1} . \Uparrow$

* See my paper on $A$ general theory of linear sets, these Transactions, vol. 27 (1925), pp. 163196. The facts summarized in this section are given in detail with proofs in that paper.

$\dagger$ Ibid., p. $164 \mathrm{ff}$.

$\ddagger$ Throughout this paper the logical symbols for implies, such that, etc., are used. Though no system of logical symbolism can yet be called classical, yet several are so similar that any one familiar with one may readily read the others. In all essentials the notation here used is the same as that used by E. H. Moore in his Introduction to a Form of General Analysis, Yale University Press, 1910. See p. 150. The definitions are also given in my paper, loc. cit., p. $163 \mathrm{ff}$.

$\S$ A general theory of linear sets, loc. cit., p. $166 \mathrm{ff}$.

|| Ibid., p. $167 \mathrm{ff}$.

Tl Ibid., p. 182 ff. 


\section{LiNEAR SYSTEMS AND TYPES OF FUNCTIONS}

In light of the results of the last section we will confine our attention to a system

$$
(R, Q, V, F)
$$

in which the elements are defined as follows.

$R$ is either the rational number system with modulus $\omega$ or a field of integers modulo a prime $p$.

$Q$ is a range; when $Q$ is the range $1,2,3, \cdots, m$ we say that $Q^{m}$.

$V \equiv$ [all $v$ on $Q$ to $R$ finitely non-zero], i. e., all functions (vectors) on $Q$ to $R$ such that there are only a finite number of elements of $Q$ for which the corresponding functional values are $\neq 0$. Obviously if $Q$ is finite $V=$ [all $v$ on $Q$ to $R$ ].

$F \equiv[$ all $f$ on $V$ to $V$ ], i. e., all functions on $V$ to $V$.

The remainder of this paper studies the solutions of $(A)$ when the $x$ 's are replaced by the $v$ 's. By ${ }_{n} C_{i} v$ we shall mean $v+v+v+\cdots\left({ }_{n} C_{i}\right.$ terms) and by $(-1)^{n}$ the element 1 of $R$ if $n$ is even and -1 of $R$ if $n$ is odd.

A function $f$ is of type $A^{k}$ if $f$ satisfies $(A)$ when $n=k$ but does not satisfy (A) if $n<k$.

We proceed to define a class of functions in $F$ which are analogous to polynomials.

Consider $H \equiv$ [all functions $h$, finitely non-zero, on $Q$ to the class of integers $1,2,3, \cdots]$. We define the weight of any $\operatorname{such} h$,

We define, letting $0^{0}=1$,

$$
w(h) \equiv \sum_{q} h(q) \text {. }
$$

$$
v^{h}=\prod_{q}(v(q))^{h(q)} \quad(v, h) .^{*}
$$

This notation is of decided convenience due to the many theorems which it suggests, for instance

$$
\text { v.). } v^{0} H=1 \text {. }
$$

Here by $0_{H}$ we mean the function in $H$ which is identically zero.

$$
\begin{aligned}
& \left.h_{1} h_{2} v .\right) \cdot v^{h_{1} h^{h_{2}}}=v^{h_{1}+h_{2}} . \\
& \quad r v h .) \cdot(r v)^{h}=r^{w(h)} v^{h} .
\end{aligned}
$$

Define

$$
{ }_{{ }_{1}} C_{h} \equiv \prod_{q}{ }_{h_{1}(q)} C_{h}(q)\left(h_{1}, h\right) ;
$$

* This notation is essentially the same as that used by E. H. Moore in his paper entitled $O n$ power series in general analysis, Mathematische Annalen, vol. 86, p. $32 \mathrm{ff}$. 
then

$$
\left.h_{1} v_{1} v_{2}\right) \cdot\left(v_{1}+v_{2}\right)^{h_{1}}=\sum_{h}{ }_{h_{1}} C_{h} v^{h} v^{h_{1}-h}
$$

The symbol ${ }_{h_{1}} C_{h}$ may be given a combinatory interpretation. Consider a class of $h_{1}(q)$ objects for each element of $Q$; then the number of ways $h(q)$ of these elements can be selected from each of the corresponding classes is the number ${ }_{h_{1}} C_{h}$, of course, using the ordinary conventions that

$$
n \text {.). }{ }_{n} C_{0}=1 \text { and } n_{1}>n_{2} \text {.). }{ }_{n_{2}} C_{n_{1}}=0 \text {. }
$$

Clearly

and

$$
\left.w(h)>w\left(h_{1}\right) .\right) \cdot h_{1} C_{h}=0
$$

$$
\text { h.). }{ }_{h} C_{h}=1 \text {. }
$$

We say that a function is of type $P^{n}$ if it is of the form

and such that

$$
\sum_{h} v_{h} v^{h}(v)
$$

$$
\begin{gathered}
\exists h: \ni: w(h)=n \cdot v_{h} \neq 0_{V}, \\
w(h)>n .) \cdot v_{h}=0_{V} .
\end{gathered}
$$

Definition of $m(h)$ :

$$
m(h)=r: \equiv: r=\text { maximum value of }(h(q)(q)) \text {. }
$$

We say that $f$ is of type $P_{n}$ if it is a function of the form

and such that

$$
\sum_{h} v_{h} v^{h}(v)
$$

$$
\begin{gathered}
\text { Э } h: э: m(h)=n \cdot v_{h} \neq 0_{V}, \\
m(h)>n .) \cdot v_{h}=0_{V} .
\end{gathered}
$$

Definition of a linear $m$-fold:

$V_{0}$ is a linear $m$-fold $: \equiv: \mathrm{H} v_{00},\left(v_{1}, \cdots, v_{m}\right)$ linearly independent

$$
\begin{aligned}
& \therefore \ni: . V_{0} \supset v: \sim: \exists r_{1} \cdot \cdot r_{m} \\
& . \ni . v=v_{00}+\sum_{i}^{1, m} v_{i} r_{i} .
\end{aligned}
$$

In such a case we say that $V_{0}=\left\{v_{00} ; v_{1}, \cdots, v_{m}\right\}$.

TheоRem 1. If $r$ belongs to $R$ and $v_{1}, v_{2}$, and $v_{3}$ belong to a linear $m$-fold $V_{0}$, then $v_{1}+r\left(v_{2}-v_{3}\right)$ belongs to $V_{0}$.

The proof is obvious. 
THEOREM 2 (the converse of Theorem 1). If for every $r$ of $R$ and $v_{1}$, $v_{2}$ of a set $V_{0}, v_{1}+r\left(v_{1}-v_{2}\right)$ belongs to $V_{0}$ and $V_{0}$ is contained in a linear $m$-fold, then $V_{0}$ is a linear $m_{1}$-fold where $m_{1} \leqq m$.

Proof. Any element of $V_{0}$ is effective as $v_{00}$ and the $v_{1}, \cdots, v_{m}$ of the definition may be readily obtained by the use of the hypothesis. Call the greatest common subset of two sets of vectors their intersection.

Theorem 3. The intersection, if it exists, of a linear $m_{1}$-fold and a linear $m_{2}$-fold is a linear $m_{3}$-fold where $m_{3}$ is equal to or less than the lesser of $m_{1}$ and $m_{2}$; or it is a vector $v$.

In light of Theorems 1 and 2 the proof is obvious.

Definition: A linear $m_{1}$-fold, $V_{1}$, and a linear $m_{2}$-fold, $V_{2}, m_{1} \geqq m_{2}$, are said to be parallel provided the difference of every two vectors of $V_{2}$ is the difference of two vectors of $V_{1}$.

In this case either $V_{1} \supset V_{2}$ or $V_{1}$ and $V_{2}$ have no intersection.

THEOREM $4 .^{*}$ If $Q$ is the finite range $(1,2,3, \cdots, m+1)$, if $V_{0}$ is a linear $m$-fold contained in the linear $(m+1)$-fold $\left\{0_{V} ; \delta_{1}, \cdots, \delta_{m+1}\right\}$ (where $\delta_{i}$ is the function $f$ for which $f(i)=1$ and $f(j)=0(i \neq j))$ then there exists uniquely $a k$ and $r_{0}, \cdots, r_{k-1}$ such that $V_{0}=\left\{v_{0} ; v_{1}, \cdots, v_{m}\right\}$ where

$$
\begin{aligned}
& v_{0}=r_{0} \delta_{k}, \\
& v_{j}=\delta_{j}+r_{j} \delta_{k} \\
& v_{j}=\delta_{j+1}
\end{aligned}
$$

THEOREM 5. Relative to $R$ with a modulus $p$, in every linear $m$-fold there exist $\left(p^{m+1}-p\right) /(p-1)$ distinct linear $(m-1)$-folds.

Proof. For each determination of $k$ in Theorem 4 there are $p^{k}$ possible sets of values for the numbers $r_{0}$ to $r_{k-1}$. Hence the number of linear $(m-1)$ folds contained in the linear $m$-fold $\left\{0_{V} ; \delta_{1}, \cdots, \delta_{m}\right\}$ is $\sum_{k=1}^{m} p^{k}=$ $\left(p^{m+1}-p\right) /(p-1)$. Clearly in light of the definition of a linear $m$-fold and Theorems 1 and 2, this carries with it the theorem for the general case.

One should note here that relative to the matrix $\left(r_{i j}\right)$ where $i$ and $j$ run from 1 to $n$, there exists for every set of vectors $v_{j}(j=1, \cdots, n)$ of $V$ a set of vectors $v_{i}^{\prime}(i=1, \cdots, n)$ such that

$$
\sum_{i}^{1, n} v_{i}^{\prime} r_{i j}=v_{j}
$$

\footnotetext{
* A general theory of linear sets, loc. cit., see p. $185 \mathrm{ff}$.
} 
if and only if the determinant $\| r_{i j}||$ does not vanish. This follows at once from the analogous classical theorem, since condition (1) may be written

$$
\sum_{i}^{1, n} v_{i}^{\prime}(q) r_{i j}=v_{j}(q)
$$

which are purely numerical conditions and for every $q$ the existence of the set $\left[v_{i}^{\prime}(q)\right](i=1, \cdots, n)$ for every set $\left[v_{j}(q)\right](j=1, \cdots, n)$ is dependent on the non-vanishing of $\left\|r_{i j}\right\|$.

IV. The solution of $(A)$. Part A

THEOREM 1. If $f$ is of type $P^{n}$ then there exists $a k \leqq n$ such that $f$ is of type $A^{k}$.

Proof. Clearly the theorem follows from the proof of it for the special case in which $f$ is of the form $\left(v_{2} v^{h}(v)\right)$, where $w(h)=n$. Clearly for any $v_{1}$ the first difference

$$
\begin{aligned}
& v_{2}\left(v+v_{1}\right)^{h}-v_{2} v^{h} \\
& \quad=v_{2} \sum_{h_{1}}^{h_{1} \pm h}{ }_{h} C_{h_{1}} v^{h_{1}} v^{h-h_{1}}
\end{aligned}
$$

is of the form $\sum_{h} v_{h} v^{h}(v)$, where $\left.v_{h} \neq 0_{V} \cdot\right) . w(h)<n$. Hence the theorem is true for $n=n_{1}$ if it is true for $n=n_{1}-1$. However we know that it holds for the case $n=0$, since in that case $f$ is a constant and the first difference vanishes. Hence by induction the proof is complete.

THEOREM 2. If $f_{1}$ and $f_{2}$ are each of the type $A^{k}, k \leqq n$, and for $n+1$ vectors of a linear 1-fold $V_{0}=\left\{v_{00} ; v_{1}\right\}$ the functional values of $f_{1}$ are equal to the functional values of $f_{2}$, then, as on $V_{0}, f_{1}$ is equal to $f_{2}$.

Proof. Clearly since the $(n+1)$ st difference vanishes Newton's interpolation formula $f\left(v_{0}+l r v_{1}\right)=v_{0}+l \Delta_{1}+\frac{1}{2} l(l-1) \Delta_{2}+\cdots$ holds for any set of values of the form $v_{00}+l r v_{1}$ for every integer $l$ and any fixed value of $r$ in $R$. Hence as on such a set $f_{1}$ is of the type $P^{k_{1}}$ and $f_{2}$ is of the type $P^{k_{2}}$, where both $k_{1}$ and $k_{2}$ are equal to or less than $n$. Hence if $p<n+1$ the theorem is vacuously fulfilled. If $p=n+1$ the theorem is obvious. If $p>n+1$ or $R$ has a modulus $\omega$ the well known theorem that a polynomial of degree equal to or less than $n$ is determined by its values for $n+1$ values of the argument carries with it our theorem since both are equivalent to the non-vanishing of the same determinant.

Part B. $Q$ Finite and $R$ With a modulus $p$

In this section we will consider $Q$ the finite range $1,2, \cdots, m$ and $R$ with a modulus $p$. 
In this section we order the elements of $R$ linearly rather than cyclically. Thus $0<1<2 \cdots<p-1$.

Theorem 1. If two functions $f_{1}$ and $f_{2}$ are each of type $P_{k_{1}}$ and $P_{k_{2}}$ respectively $\left(k_{1} \leqq k_{2} \leqq n<p\right)$, and $V_{1}=$ [all vectors of the form $\left.\sum_{i=1}^{m} r_{i} \delta_{i}\left(r_{i} \leqq n(i)\right)\right]$ and, as on $V_{1}, f_{1}=f_{2}$, then $f_{1}=f_{2}$ and corresponding coefficients are equal.

Proof. This theorem is, of course, equivalent to the theorem that if $f$ is of type $P_{k}(k \leqq n<p)$ and if $f$ as on $V_{1}$ is identically 0 then $f$ is identically $0_{V}$ and all the coefficients are $0_{V}$.

Consider the coefficients of the polynominal in $r_{1}$ which are themselves polynomials in $r_{2}$ to $r_{m}$ with coefficients in $V$ which are of degree equal to or less than $k$ in $r_{2}$ to $r_{m}$ and vanish for all values of $r_{2}$ to $r_{m}$ less than or equal to $n$, since the polynomial vanishes for $n+1$ values of $r_{1}$ whenever $r_{2}$ to $r_{m}$ are all equal to or less than $n$; hence we have a reduction process and every coefficient must be $0_{V}$.

THEOREM 2. If $V_{1}=$ [all vectors of the form $\left.\sum_{i=1}^{m} r_{i} \delta_{i}\left(r_{i} \leqq n(i)\right)\right]$ where $n<p$, and $g$ is a function on $V_{1}$ to $V$, then there exists one and only one function $f$ of type $P_{k}(k \leqq n)$ such that $f\left(\right.$ as on $\left.V_{1}\right)=g$.

Proof. There are $p^{m(n+1)^{m}}$ distinct functions of type $P_{k}(k \leqq n)$ and since by Theorem 1 these functions as on $V_{1}$ are distinct they must as on $V_{1}$ be the $p^{m(n+1)^{m}}$ distinct functions on $V_{1}$ to $V$.

Hence every function $f$ on $V$ to $V$ is of type $P_{k}$ where $k<p$. Every function is, therefore, of type $P^{l}$ where $l \leqq(p-1) m$.

TheOREM 3. If $f$ is of type $A^{k}(k<p-1)$ then $f$ is of type $P^{k}$ and conversely.

Proof. $f$ is of type $P_{l}$ where $l<p$, i. e. $f$ is of the form $\sum_{h} v_{h} v^{h}$ where $m(h)>p-1$.) $\cdot v_{h}=0_{V}$. As on any linear 1 -fold with typical element $v_{0}+r v_{1}$, Newton's interpolation formula shows that $f$ is a polynomial in $r$ with coefficients in $V$ of degree less than or equal to $k$. By substitution of $v=v_{0}+r v_{1}$ $i t$ is seen that

$$
\begin{aligned}
f & =\sum_{h} v_{h}\left(v_{0}+r v_{1}\right)^{h} \\
& =\sum_{h} v_{h} \sum_{h_{1}}{ }_{h} C_{h_{1}} v_{0}{ }^{h-h_{1}} r{ }^{\left(h_{1}\right)} v_{1}^{h_{1}} \\
& =\sum_{h} v_{h} \sum_{k_{1}}^{w\left(h_{1}\right)} \sum_{h_{1}}^{k_{1}(p-1)}{ }_{h} C_{h_{1}} v_{0}^{h-h_{1}} r^{w\left(h_{1}\right)} v_{1}^{h_{1}} .
\end{aligned}
$$

Thus, since for any integer $\alpha, \gamma^{\alpha}=r^{\alpha+p-1}$,

$$
\left.\left.p>k_{1}>k .:\right): v_{0} \cdot v_{1}:\right): \sum_{h}{ }^{w\left(h_{1}\right)} \sum_{h_{1}}^{(p-1)} v_{h h} C_{h_{1} v_{0}}{ }^{h-h_{1}} v_{1}^{h 1}=0_{V} \text {. }
$$


Hence, since this holds for all $v_{1}$ by Theorem 1 ,

But this holds for every $v_{0}$, so

$$
\left.\left.p>k_{1}>k .:\right):: v_{0} \cdot w\left(h_{1}\right) \equiv k_{1}(p-1):\right): \sum_{h} v_{h h} C_{h_{1}} v_{0}^{h-h_{1}}=0_{V} \text {. }
$$

Moreover,

$$
\left.\left.h \cdot p>k_{1}>k .:\right): . w\left(h_{1}\right) \equiv k_{1}(p-1) .\right) . v_{h h} C_{h_{1}}=0_{V} \text {. }
$$

$$
\left.p>k_{1} \cdot h: \ni: m(h)<p \cdot w(h) \geqq k_{1} .:\right): . \exists h_{1}: \ni: w\left(h_{1}\right) \equiv k_{1}(p-1) \cdot{ }_{h} C_{h_{1}} \not \equiv 0(p) .
$$

Hence,

$$
p-1>k \cdot w(h)>k:): v_{h}=0_{V} .
$$

Therefore, if $f$ is of type $A^{k}$ it is of type $P^{l}$ where $l \leqq k$, but, if $l<k$, $f$ would be of type $A^{l_{1}}\left(l_{1} \leqq l\right)$ (see Part A, Theorem 1) which is in contradiction to our hypothesis and, hence, $f$ is of type $P^{k}$. The converse follows at once in light of Theorem 1 of Part A.

\section{PART C. $Q$ Finite AND $R$ Rational}

In this part we will consider $Q$ a finite range and $R$ isomorphic with the field of all rational numbers.

In order to pass from the case where $R$ has a modulus $p$ to the case in which $R$ is isomorphic with the field of rational numbers one should note the following theorem.

TheOREM 1. Relative to an $m$ by $m$ non-singular matrix $u=\left(r_{i j}\right)$ whose elements are rational integers, and $a$ set of integers $c_{i}(i=1, \cdots, m)$ there exists a proportionality factor $\lambda$ and an integer $n$ such that the solution of the equations

$$
\sum_{j} r_{i j} x_{j}=\lambda c_{i}
$$

is the reduced solution of the congruences

for every $p>n$.

$$
\sum_{j} r_{i j} x_{j} \equiv \lambda c_{i}(p)
$$

By reduced solution is meant the solution for which each $x$ is in absolute value equal to or less than $p / 2$.

Proof. The system of linear equations

$$
\sum_{j} r_{i j} x_{j}=c_{i}\|\mu\|
$$

may be reduced by multiplication by integers (cofactors of the elements of $\|\mu\|)$ and addition to the system 


$$
\|\mu\| x_{j}=\Delta_{j}\|\mu\|
$$$$
(j=1,2, \cdots, m)
$$

where $\Delta_{j}$ is the determinant of the matrix secured by replacing the $j$ th column of $\mu$ by the $c$ 's. If $n$ is greater than twice the absolute value of any of the multipliers or the coefficients of the unknowns in the equations of this reduction process, then for $p>n$ the reduction in the sense of a congruence modulo $p$ would be formally identical. Since $n$ is chosen greater in absolute value than $2\|\mu\|, 2 \Delta_{j}$ and $2 \Delta_{j}\|\mu\|$, for every $j$, the reduced solution of the congruence is $x_{j}=\Delta_{j}$.

$$
\|\mu\| x_{j} \equiv \Delta_{j}\|\mu\|(p) \text { where } p>n
$$

Hence $\|\mu\|$ is effective as $\lambda$.

THEOREM 2. If on $V_{1}=$ [all vectors of the form $\sum_{i=1}^{m} r_{i} \delta_{i}\left(0 \leqq r_{i} \leqq n\right.$ and integers) (i)] two functions $f_{1}$ and $f_{2}$ of type $A^{k}(k \leqq n)$ are equal, the two functions are equal.

Proof. This can be readily arrived at by repeated application of Newton's interpolation formula. Obviously if $f_{1}$ is determined on $V_{1}$ it is determined for all vectors such that $v(i)$ is an integer or 0 , less than or equal to $n$ if $i \neq 1$ and unrestricted as to the value of $v(1)$. By another application the restriction on $v(2)$ may be removed, etc.

Moreover, from consideration of Newton's interpolation formula, if, as on $V_{1}, f_{1}$ has integral values for its coördinates, it has integral values for its coördinates for all $P$ whose coördinates are integers.

TheOREM 3. If $g$ is of type $A^{k}(k \leqq n)$ then there exists one and only one function $f$ of type $P_{l}(l \leqq n)$ such that $f$ (as on $\left.V_{1}\right)=g$ (as on $\left.V_{1}\right)$.

Proof. This theorem holds provided the $(n+1)^{m}$ by $(n+1)^{m}$ matrix of the system of equations for the coefficients of the terms of $f$

$$
\sum_{h} v_{h} v^{h}=g(v) \quad\left(v \text { in } V_{1}\right),
$$

summation extending over all $h$ such that $m(h) \leqq n$, is non-singular.

But this matrix reduced modulo $p \geqq n$ is non-singular since Theorem 2, Part B, holds.

Since $g$ is of type $A^{k}(k \leqq n)$, there exists a $\lambda$ such that $g_{1} \equiv \lambda g$ has integral coördinates for all vectors $v$ whose coördinates are integers. If for all vectors $v$ with integral coördinates we reduce the coördinates of $g_{1}(v)$ modulo $p$ $\left(p>n\right.$ ) we have a new function $g_{1}^{\prime}$ (on all $v$ with integral coördinates to all $v$ with integral coördinates). 
Moreover, $g_{1}^{\prime}$ is of type $A^{k_{1}}\left(k_{1} \leqq k\right)$ and has the property that for every $v_{1}$ and $v_{2}$ with integral coördinates $g_{1}^{\prime}\left(v_{1}+p v_{2}\right)=g_{1}^{\prime}\left(v_{1}\right)$. Hence $g_{1}^{\prime}$ can be used to define a function of type $A^{k_{1}}$ in the case where $R$ has a modulus $p$. Moreover, if $p$ is chosen sufficiently large, $k_{1}=k$, since there will exist some values of $v_{0}$ and $v_{1}$ relative to which equation $(A)$ holds for $n=k$ but not for $n=k-1$ and if $p$ is sufficiently large the same will be true for $g_{1}^{\prime}$.

Hence by Theorem 1 and Theorems 2, 3, Part B, if $p$ is sufficiently large the solution of equation (1) with $g$ replaced by $g_{1}^{\prime}$ yields a function of type $P^{k}$ and is the same as the solution of (1) with $g$ replaced by $g_{1}$. But $g_{1}$ differs from $g$ only by a constant factor. Hence $f$ is of the type $P^{k}$.

But by Theorem 1, Part A, $f$ is of type $A^{k_{2}}\left(k_{2} \leqq k\right)$ and therefore by Theorem 2 of this part $f=g$. Hence we may conclude

THEOREM 4. If $V$ is the set of all vectors on a finite range $Q$ to $R$ then any function of type $A^{n}$ is of type $P^{n}$ if $R$ is isomorphic with the field of all rational numbers or has a modulus $p>n+1$. If $p=n+1$ then $f$ is of type $P^{k}$ where $k \geqq n+1$ and $P_{l}$ where $l<p$. There are no functions of type $A^{k}$ where $k \geqq p$.

Note: Another proof of the above theorem for the case in which $R$ is rational exhibits a set of vectors such that for every corresponding set of values there exists one and only one function of type $A^{k}(k \leqq n)$ having for these vectors the assigned functional values. This proof is inserted here for the added insight it gives into the general problem of setting up solutions of equation $(A)$ although as a proof of the above theorem it is much longer than the first one given.

Let ${ }_{n} E_{m}$ be the number of ways that $n$ or any integer less than $n$ may be expressed as the sum of $m$ integers or zeros, regarding each change of order as a new way. ${ }_{n} E_{m}$ is also the number of distinct vectors $h$ on the range $1,2,3, \cdots, m$ to the range $0,1,2,3, \cdots$ such that $w(h) \leqq n$ and hence is the maximal number of non-zero terms in a polynomial of degree $n$ in $S_{m}$, that is, the maximum number of non-zero terms in a function of type $P^{n}$ when $Q^{m}$.

It is readily shown that

$$
{ }_{n} E_{m}=\sum_{i}^{0, n}{ }_{i} E_{m-1} .
$$

Consider a set $E$ of ${ }_{n} E_{m}$ vectors arranged in $n+1$ linear $(m-1)$-folds $S_{0}, S_{1}, \cdots, S_{n}$, such that every pair $S_{i}, S_{j}(i \neq j)$ intersect in a linear $(m-2)$ fold and these linear $(m-2)$-folds are pair by pair non-parallel, and such that no three intersect in the same $(m-2)$-fold. Let $S_{0}$ contain a set $E_{0}$ of ${ }_{n} E_{m-1}$ vectors of $E$. Let $S_{1}$ contain a set $E_{1}$ of ${ }_{n-1} E_{m-1}$ vectors of $E$ not 
in $S_{0}$. Let $S_{2}$ contain a set $E_{2}$ of ${ }_{n-2} E_{m-1}$ vectors of $E$ not in $S_{0}$ or $S_{1}$, etc. Moreover, let the ${ }_{n-i} E_{m-1}$ vectors of $E_{i}$ be arranged in $n-i+1$ linear $(m-2)$ folds $S_{i 0}, \cdots, S_{i n-i}$ contained in $S_{i}$ and not parallel to the intersection of any $S_{i}(j<i)$ with $S_{i}$ and such that pair by pair they intersect in nonparallel linear $(m-3)$-folds and such that $S_{i 0}$ contains a set $E_{i 0}$ of ${ }_{n-i} E_{m-2}$ vectors of $E_{i}, S_{i 1}$ contains a set $E_{i 1}$ of ${ }_{n-i-1} E_{m-2}$ vectors of $E_{i}$ not in $S_{i 0}$, etc. Continue this mode of subdivision until all vectors of $E$ are distributed in linear 1 -folds.

The theorem that relative to any function $g$ on $E$ to $V$ there exists one and only one function $f$ of type $P^{k}(k \leqq n)$ such that, as on $E, f=g$, is equivalent to the non-vanishing of the determinant $D$ of the matrix $\mu=\left(r_{v h}\right)$ where $r_{v h}=v^{h}$ and $v$ runs through the ${ }_{n} E_{m}$ vectors of $E$ while $h$ runs through the ${ }_{n} E_{m}$ vectors on the range $1,2,3, \cdots, m$ to the integers and 0 such that $w(h) \leqq n$.

Clearly by a reversible transformation $S_{0}$ may be taken as the linear $(m-1)$-fold $\left\{0_{V} ; \delta_{1}, \cdots, \delta_{m-1}\right\}$. Hence, the minor corresponding to $E_{0}$ and the set $H_{0}$ of $H$ such that, for any vector $h_{0}$ of $H_{0}, h_{0}(m) \neq 0$, is composed of zero elements only. Hence $D$ is the product of the determinant $D_{1}$ of the minor $\mu_{1}$ of $\mu$ which has elements $r_{v h}$ such that $v \subset E_{0}$ and $h(m)=0$ and the determinant $D_{2}$ of the minor $\mu_{2}$ which has elements $r_{v h}$ such that $v$ is not contained in $E_{0}$ and $h(m) \neq 0$.

The non-vanishing of $D_{1}$ is the condition that the theorem holds for $Q_{1}=1,2, \cdots, m-1$.

$D_{2}=\left(\prod_{v} v(m)\right) D_{3}$, where the product extends over all $v$ in $E$ not in $E_{0}$ and hence not in $S_{0}$ and hence is different from zero, whereas the nonvanishing of $D_{3}$ is equivalent to our theorem for the case of order $n-1$.

Hence by a finite number of steps we can reduce our theorem to the two special cases

(1) the case in which $Q$ is singular;

(2) the case in which $n=0$.

In the first case the theorem is equivalent to the fact that a polynomial of degree less than $n$ is determined by its value for $n+1$ values of the argument. In the second case $f$ is a constant and the theorem is obvious.

If this work is to lead to an independent proof of Theorem 3 we should note that if two functions $f_{1}$ and $f_{2}$ are of type $A^{k}(k \leqq n)$, and, as on $S_{0}, \cdots$, $S_{n}, f_{1}=f_{2}$, then $f_{1}=f_{2}$. For consider any vector $v$ not in any of the sets $S_{0}, \cdots$, $S_{n}$; then there exists a linear 1 -fold $V_{0}$ containing $v$ and $n+1$ distinct vectors $v_{0}, \cdots, v_{n}$ such that $S_{i} \supset v_{i}$. Hence, by Theorem 2 , as on $V_{0}, f_{1}=f_{2}$ and hence $f_{1}(v)=f_{2}(v)$ for every $v$, i. e., $f_{1}=f_{2}$. However, the determination of a function of type $A^{k}(k \leqq n)$ on $S_{0}$ depends, in a similar manner, on the determination 
of the function on $S_{00}, \cdots, S_{0 n}$, the determination of the function on $S_{1}$ depends on its determination on $S_{10}, \cdots, S_{1 n-1}$ and on the intersection of $S_{0}$ and $S_{1}$; its determination on $S_{2}$ depends on its determination on $S_{20}, \cdots, S_{2 n-2}$ and the intersection of $S_{2}$ with $S_{1}$ and $S_{0}$; hence, we reduce the case for each linear $m$-fold considered to the case for $n+1$ linear $(m-1)$ folds and finally to the vectors of $E$. Hence any function of type $A^{k}(k \leqq n)$ must be identical with the function of type $P^{k}$ having the same functional values on $E$.

This proof does not hold in general for the case where $R$ has a modulus $p$ a prime, as can be readily seen from the Theorem 5 of $\S$ III.

\section{Part D. $Q$ General}

In this part we consider $Q$ as a general range.

We shall show that any solution of $(A)$ is of the form

$$
\sum_{h} v_{h} v^{h}
$$

For any $h_{1}$ consider the set $Q_{h_{1}}=\left[\right.$ all $\left.q .{ }^{\prime} . h_{1}(q) \neq 0\right] ; Q_{h_{1}}$ is finite. Consider $Q_{1}$ and $Q_{2}$, finite subsets of $Q$ such that $Q_{1} \supset Q_{2} \supset Q_{h_{1}}$. By the theorem of the last section, $f$ as on $Q_{1}$ is of the form

and as on $Q_{2}$ is of the form

$$
\sum_{h} v_{1 h} v^{h}
$$

$$
\sum_{h} v_{2 h} v^{h}
$$

From the unique determination of the $v$ 's as shown in the above sections it is readily seen that $v_{1 h_{1}}$ (as on $\left.Q_{2}\right)=v_{2 h_{1}}$, since $v_{1 h_{1}}$ as on $Q_{2}$ must be completely determined by the value of $f$ for $v$ 's for which $v(q) \neq 0.) . Q_{2} \supset q$. Let the set $Q_{2}$ swell. $^{*}$ It is readily seen that a $v_{h_{1}}$ is determined uniquely as a limit. Consider, using the above determined $v$ 's, the function

$$
\sum_{h} v_{h} v^{h}
$$

we readily see that it is identical with $f$, since for any $v$ all terms except those for which $Q_{h} \subset$ [all $q . \ni . v(q) \neq 0$ ] vanish and, for any such $h, v_{h}$ as on $Q_{v}$ is the same on any finite range containing $Q_{v}$ as the coefficient we

* The consideration of this kind of limit has been given by E. H. Moore in his courses in General Analysis and a theory of limits including this as a special case has been discussed by E. H. Moore and H. L. Smith in their joint paper entitled $A$ general theory of limits, A merican Journal of Mat hem a tics, vol. 44 (1922). 
would arrive at in the finite case. Moreover, if $f$ is of the type $A^{n}$ and $R$ has a modulus $\omega$ or $p>n+1$ then $f$ is of type $P^{n}$, but if $p=n+1$ we can only conclude that $f$ is of type $P_{k}(k \leqq n)$ and that there exists an $h$ such that $w(h) \geqq n, v_{h} \neq 0$.

V. Certain SOlutions DiRECTLy In TERMS OF A NUMBER SYSTEM $\mathfrak{A}$ OF TYPE $A$ Returning to a number system $\mathfrak{A}$ of type $A$ and considering functions on $\mathfrak{A}$ to $\mathfrak{A}$ the following theorem holds.

THEOREM 1. A function $f$ of the form

$$
\sum_{k}^{0, n} a_{k 0} \prod_{i}^{1, k}\left(a a_{k i}\right) \quad(a),
$$

where $\prod_{i=0}^{n} a_{n i} \neq 0$, is of the type $A^{l}$ where $l \leqq n$.

Proof. Consider a function of the form

$$
a_{k 0} \prod_{i}^{1, k}\left(a a_{k i}\right)(a), \text { where } \prod_{i}^{0, k} a_{k i} \neq 0
$$

The first difference, for a difference $a_{1}$ in the argument, is

$$
a_{k 0} \prod_{i}^{1, k}\left(a+a_{1}\right) a_{k i}-a_{k 0} \prod_{i}^{1, k}\left(a a_{k i}\right) \quad(a) .
$$

Clearly this function is of the form (1) where $n<k$, or is 0 . Hence the theorem follows by induction.

\section{Continuous solutions}

There is particular interest in the case where $\mathfrak{A}$ contains a field $\mathfrak{R}$ isomorphic with the real number system and commutative with $\mathfrak{A}$. It can be shown* that there exists a range $Q$ such that $\mathfrak{A}$ is isomorphic with the set $V$ of all finitely non-zero vectors on $Q$ to $\Re$. If one defines the "écart" or distance of any two vectors $v_{1}$ and $v_{2}, \epsilon\left(v_{1}, v_{2}\right)$, as the maximum of the absolute value of the functional values of their difference, one can readily define (using the usual definition) a continuous function $f$ on $V$ to $V$ thus:

$$
\begin{gathered}
f C: \equiv: f:: \ni:: v, e>0 .:): . \mathrm{G} r_{e v}>0: э: \epsilon\left(v, v_{1}\right)<r_{e v} \\
.) . \epsilon\left(f(v), f\left(v_{1}\right)\right)<e .
\end{gathered}
$$

Since by Newton's interpolation formula any solution of $A$ is for any linear 1-fold $\left\{v_{0} ; v_{1}\right\}$ a polynomial in $r$ as on all values of the form $v_{0}+v_{1} r$,

* A general theory of linear sets, loc cit., p. $182 \mathrm{ff}$. 
where $r$ is rational, then any continuous solution will be a polynomial as on $\left\{v_{0} ; v_{1}\right\}$.

Moreover, in considering the case in which $Q^{m}$ it is readily seen that any continuous solution is determined if the solution is determined for all $v$ such that the functional values of $v$ are rational. Hence, one may readily see from the results of the preceding sections

THEOREM 1. Any continuous function of type $A^{n}$ is a function of type $P^{n}$ (where the terms are now understood to be relative to the $Q, \Re, V$ system).

To feel the force of the last theorem the following three corollaries are worth noting.

Corollary 1 . If $\mathfrak{A}$ is the real number system any continuous function of type $A^{n}$ is a polynomial of degree $n$.

This, of course, is obviously provable without reference to the theorem.

CoROLlary 2. If $\mathfrak{A}$ is the complex number system any continuous function of type $A^{n}$ is a polynomial of degree $n$ in the coördinates $x$ and $y$ of $z=x+i y$, the coefficients of which are complex numbers.

COROLLARY 3. If $\mathfrak{A}$ is the system of real quaternions any continuous function of type $A^{n}$ is a polynomial of degree $n$ in the coördinates $x, y, z$, and $w$ of $q=x+i y+j z+k w$, in which the coefficients are quaternions.

UNIVERSITY OF WISCONSIN, Madison, Wis. 